\title{
MOBILE SNIFFER AND JAMMER
}

\author{
Sujith $\mathbf{M}^{\mathbf{1}}$, Bibin Joseph ${ }^{2}$, Anoop P S ${ }^{3}$, Dileep John ${ }^{4}$ \\ ${ }_{1,2,3,4}$ B.Tech student, ECE, MBITS, Nellimattom, Kerala, India
}

\begin{abstract}
This system detects the use of GSM mobiles in mobile restricted areas such as examination halls, hospitals and other do not disturb areas. The sniffer keeps monitoring the RF level in the area and gives a warning indication if the RF level increases due to mobile activities. This warning indication is send as a message through a GSM module and displayed on a LCD. The pic 16F877A Microcontroller contains internal flash memory of $8 \mathrm{~kb}$ along with a large RAM area and internal EEPROM. To make it fit for monitoring and real time system applications microcontroller is induced with an 8 channel 10 bit A/D converter. Liquid crystals are materials that have a molecular structure and will flow like a liquid. It is also a low power device. Some properties of this molecular structure are associated with solids. An antenna whose receiving or radiating elements, such a arms of a dipole, are made in the form of an extensible system of metal tubes or rods of approximately equal length. Such a design facilities changing the length of antenna elements during tuning and permits the size of antenna to be reduced when it is not in operation for example during transport or storage. Telescopic antennas are used principally in conjunction with radio receivers, radio transmitter-receivers, portable television receivers and television receivers installed in moving objects. To make it cost effective and to have a small dimension SIM Com is induced with in a powerful single chip processor integrating AMR 926EJ-S core and quad band-GSM/GPRS module of SMT type. SIM Com contains a reliable wireless module-SIM900 and is ultra compact.
\end{abstract}

Keywords: Rooting, Sniffer, Dipole.

\section{INTRODUCTION}

Mobile sniffer with alert system is designed as a sensitive RF detector. This circuit detects the use of GSM mobiles in mobile restricted areas such as examination halls, hospitals and other 'do not disturb' areas.

The sniffer circuit consists of an RF detector, Peripheral Interface Controller (PIC), and a GSM module. The PIC used in this circuit is PIC16F877A and the GSM module used is SIM900. A telescopic areal is used as the receiving antenna. An LCD display is used as an alternative indication.

\section{BLOCK DIAGRAM}

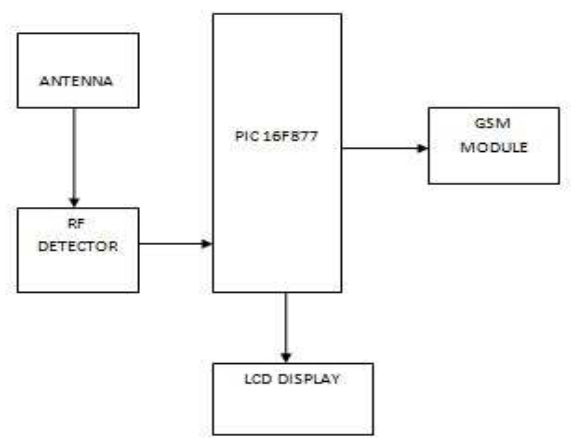

Fig -1: Block Diagram

\subsection{Block Diagram Explanation}

\subsubsection{PIC 16F877A Controller}

The PIC 16F877A Microcontroller contains internal flash memory of $8 \mathrm{~kb}$ along with a large RAM area and internal EEPROM. To make it fit for monitoring and real time system applications microcontroller is induced with an 8 channel 10 bit A/D converter. In order to avoid the removal of microcontroller the board is enabled with updating of new codes by making the circuit program to be downloaded to make the ports connect and disconnect easily standard headers are brought.

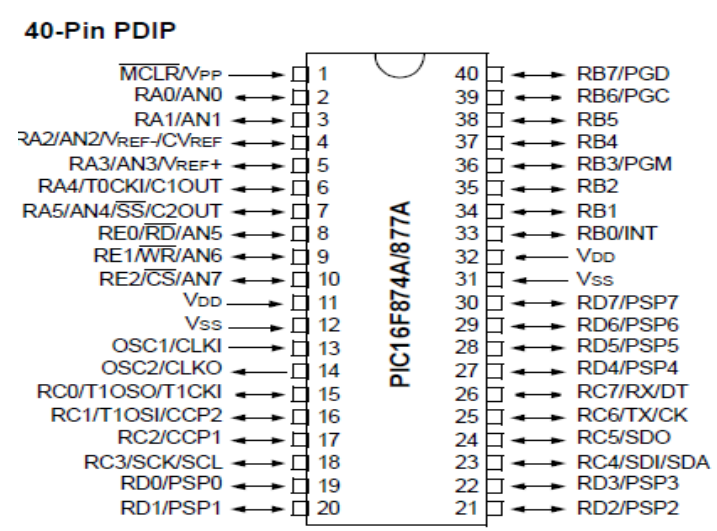

Fig -2: PIC16F877 Microcontroller 


\subsubsection{LCD Display}

Liquid crystals are materials that have a molecular structure and will flow like a liquid. It is also a low power device. Some properties of this molecular structure are associated with solids. Additional to the power requirement of an LCD ( in the order in the of microwatts) it also requires an external and internal light source The power requirement is typically in the order of microwatts for the LCD. As LCD's are prone to chemical degradation its temperature range is limited to zero to 60 degree Celsius and lifetime is a major concern.

LCD's are mainly classified into:

1. Dynamic-scattering LCDs

2. Field- effect LCDs

When we consider source of energy as a prime factor field effect LCDs are normally used (e.g. timers, portable instrumentation etc.). Apart from scattering type, they absorb less power source but their cost is typically higher and the height is limited to 2 inches, where as light scattering units are available to 8 inches in height.

The turn-on and turn-off time is an important consideration in all displays. The response time of LCDs is in the range of 100 to $300 \mathrm{~ms}$. The lifetime of LCDs is steadily increasing beyond 10000+hours limit.

\subsection{Telescopic Antenna}

An antenna whose receiving or radiating elements, such as arms of a dipole, are made in the form of an extensible system of metal tubes or rods of approximately equal length. Such a design facilitates changing the length of the antenna elements during tuning and permits the size of the antenna to be reduced when it is not in the operation-for example, during transport or storage. For each pairs of telescoping tubes, interior diameter of the outer tube is approximately equal to exterior diameter of the inner tube. The tubes fit into one another with some friction, which is needed to provide electrical contact and to maintain the required length of each elements of the telescopic antenna under operating conditions-i.e., under the action of gravity or exposure of shaking or vibration. In some cases, the required position of the tube may be fixed by additional means.

Telescopic antennas are used principally in conjunction with radio receivers, radio transmitter-receivers, portable television receivers and television receivers installed in moving objects.

\subsection{GSM Module(SIM 900)}

To make it cost effective and to have a small dimension SIM Com is induced with in a powerful single chip processor integrating AMR 926EJ-S core and quad band-GSM/GPRS module of SMT type
The SIM900 offers GSM/GPRS 850/900/1800/1900MHz performance for voice, SMS, Data, and Fax in a small form factor and with low power consumption as per industrial standard interface. Due to its small dimension $(24 \mathrm{~mm} \times 24 \mathrm{~mm}$ x $3 \mathrm{~mm}$ ) it can almost fit into M2M applications and also for slim and compact demands of design.

\subsection{General Features}

*Quad-Band 850/ 900/ 1800/ 1900 MHz*GPRS multislot class $10 / 8$

*GPRS mobile station class B

*Compliant to GSM phase $2 / 2+$

- Class 4 (2 W @ 850/900 MHz)

- Class 1 (1 W @ 1800/1900MHz)

*Dimensions:24*24*3mm

*Weight:3.4g

* Control via AT commands (GSM $07.07,07.05$ and

SIMCOM enhanced AT Commands)

*SIM application toolkit

* Voltage source range : $3.2 \ldots 4.8 \mathrm{~V}$

* Power consumption is low: $1.0 \mathrm{~mA}$ (sleep mode)

* Working temperature range: $-40^{\circ} \mathrm{C}$ to $+85^{\circ} \mathrm{C}$

\section{CIRCUIT DIAGRAM}

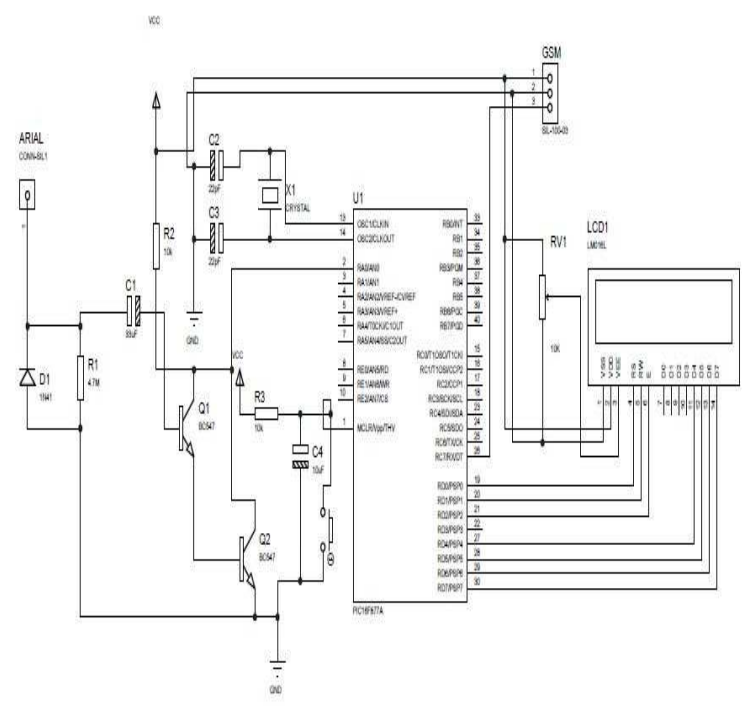

Fig-3 : Circuit Diagram

\subsection{Circuit Description}

The RF detector diode detects the change in RF signal level and produces the corresponding voltage change. This change in voltage is given to digital to converter pin of a PIC, which reads the value of ADC due to a change in voltage when mobile is called. Whenever the voltage changes a message is send by the GSM module indicating the detection of mobile usage. An LCD is used to display the message send. 


\section{PCB DESIGN AND FABRICATION}

\subsection{PCB Design}

PCB design of the circuit was implemented using DIP TRACE. Initially the schematic of the circuit was drawn. The necessary PCB foot prints were given, thereby specifying the component size and spacing. After giving the foot prints a new list was created.

Rooting procedure was done using DIPTRANCL PCB LAYOUT software. The net list was loaded and components spacing done accordingly. Using the auto routing potion, rooting was done. The possible connections were made using auto rooting and remaining connections were done manually using jumpers.

\subsection{PCB Fabrication}

After the PCB design phase, PCB fabrication was done the path was drawn on a circuit board using a carbon paper and the mirror image obtained was painted. The board was etched by dipping it in a highly concentrated ferric chloride and after etching, the remaining paint was removed. The continuity of all paths $\mathrm{w}$ as checked and then the components were placed and soldered. After providing the necessary jumpers also the circuit was tested.

\subsection{Soldering}

The process of joining two metals to have physical bonding and electrical conductivity is called soldering. Soldering is mainly used to build electronic circuits. Soldering is done by using a soldering iron which consists of a heating element, solder tip, power cord. A resistive element is used as the heating element.

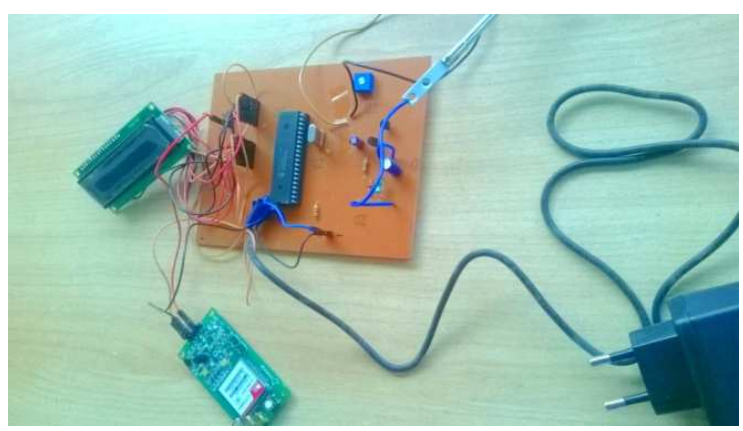

Fig-4 : Mobile sniffer

A specially shaped piece of copper plated with iron and then usually plated with chrome or iron is the soldering bit. A wide variety of voltages such as $12 \mathrm{v}, 24 \mathrm{v}, 115 \mathrm{v}$, and $230 \mathrm{v}$ are available. Low voltages should be used for safer soldering. Advanced soldering works require temperature control which is usually set between 200 and 450 degree celcius. For quick repairs gas powdering soldering iron are used.
Some of the soldering fluxes may be dangerous. A smell fan or fume top can be used to avoid risk. The room must be ventilated, fume extraction system can be used in professionally electronic workshop to protect workers. Hand should be washed properly after soldering. Turn off the soldering iron when it is not used.

\section{PCB LAYOUT}

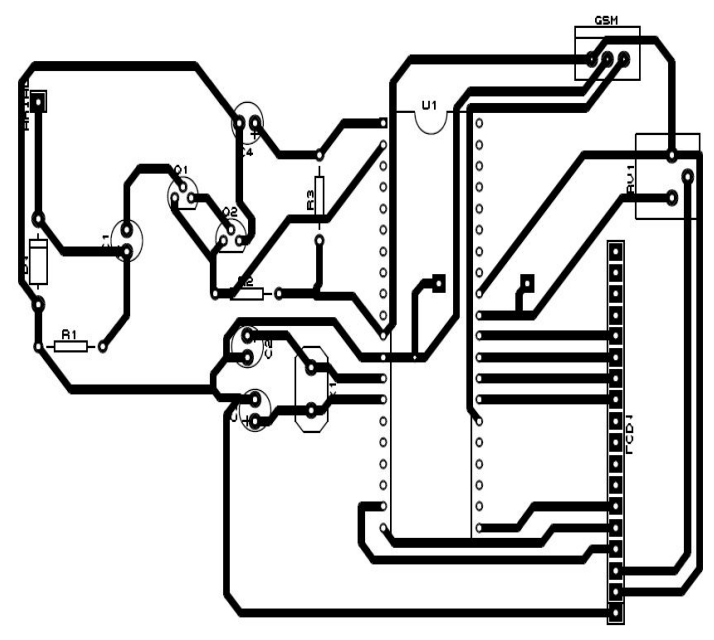

Fig-5: PCB Layout (Bottom view)

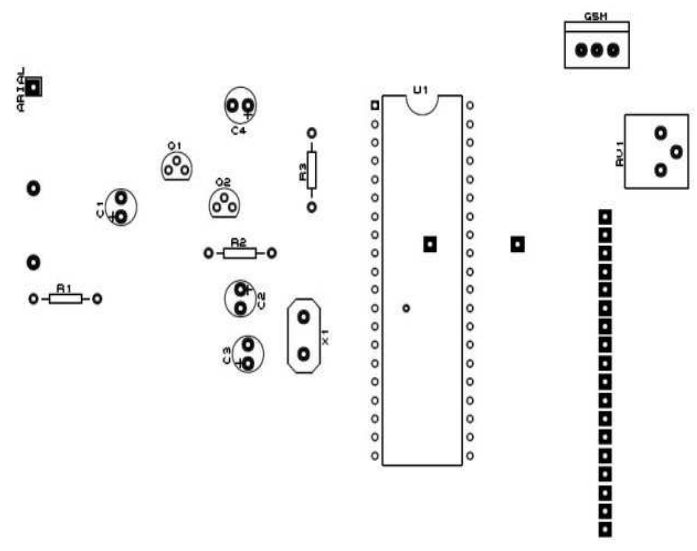

Fig-6 : PCB Layout (Top view)

\section{RESULTS}

The mobile sniffer with alert system project was successfully completed and desired results are obtained. The final product is a sniffer circuit which continuously monitor the RF level in area. If the RF level increases due to the use of a mobile, the sniffer circuit detects the use and a warning message is send to the controller by using a GSM module and the message is displayed through a LCD display. The working of the device was tested and demonstrated successfully. 


\section{FUTURE ENHANCEMENT}

As we all know that the use of mobile phones has been increasing from place to place for the past few years. Mobile phone has been very useful to us in this technological world. But there are places where mobile phones are restricted. To detect the use in the restricted areas we use mobile sniffer. Some of the restricted are hospitals, schools, colleges, examination halls etc. Mobile sniffer with alert system are using in some part of the world and in future it will be dominant.

\section{CONCLUSIONS}

There are certain boundary conditions that have to be qualified for the identification of lost mobiles like the power of the mobile should be good enough, the mobile phone should not in the shadow region but however this method using modern technologies and devices.

\section{REFERENCES}

[1]. Schiller,"Mobile Communication",Pearson Education, 1 Edition,7th reprint-2003

[2]. John D Kraus,"Electromagnetic",TMH

[3]. Jordan et al,"Electromagneticwaves and radiation system",Printice Hall

[4]. www.gsmworld.com

\section{BIOGRAPHIES}

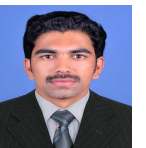

SUJITH M, Presently doing final year B tech graduation in ECE from MG University. Like to do research in Netwoking and communication.

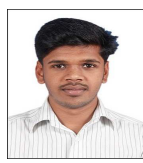

BIBIN JOSEPH, Presently doing final year B tech graduation in ECE from MG University. Like to do research in Digital Electronics.

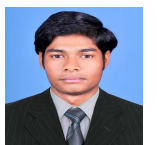

ANOOP P S, Presently doing final year B tech graduation in ECE from MG University. Like to do research in Electromagnetic theory.

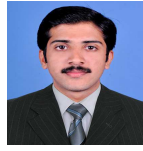

DILEEP JOHN, Presently doing final year B tech graduation in ECE from MG University. Like to do research in Electromagnetic theory and waveguides. 